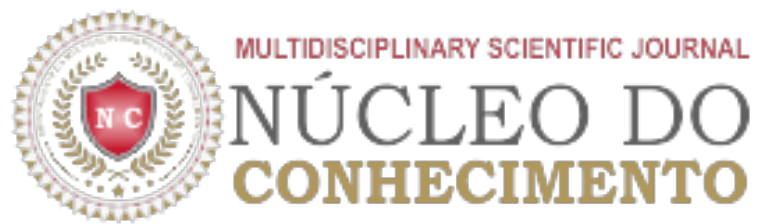

\section{Auditoria de Enfermagem na Qualidade da Assistência à Saúde Pública}

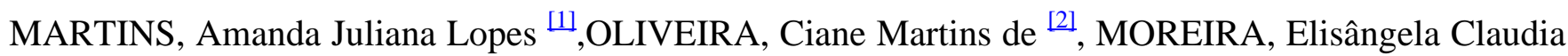
de Medeiros ${ }^{[3]}$, FECURY, Amanda Alves ${ }^{[4]}$, DIAS, Cláudio Alberto Gellis de Mattos ${ }^{[5]}$, DENDASCK, Carla Viana ${ }^{[6]}$, OLIVEIRA, Margaret de ${ }^{[7]}$,OLIVEIRA, Euzébio de ${ }^{[8]}$

MARTINS, Amanda Juliana Lopes; et.al. Auditoria de Enfermagem na Qualidade da Assistência à Saúde Pública. Revista Científica Multidisciplinar Núcleo do Conhecimento. Ed. 11, Ano 02, Vol. 04. pp 96-113, Novembro de 2017. ISSN:2448-0959

\section{RESUMO}

O presente estudo teve como objetivo conhecer a importância da auditoria de enfermagem nos serviços de saúde pública e compreender sua influência direta na qualidade da assistência prestada, na redução dos prejuízos financeiros, na utilização inadequada de recursos e em um melhor controle de gastos. Este artigo foi realizado através de uma revisão bibliográfica onde foram consultadas produções publicadas no período correspondente aos últimos quinze anos. A busca desse material foi realizada na Biblioteca Virtual da Saúde (BVS), tendo como base de dados: a Scientific Eletronic Library Online-Brasil (SCIELO), na revista eletrônica de enfermagem e no Google acadêmico, onde foram utilizados os seguintes descritores: auditoria, auditoria de enfermagem, auditoria em saúde pública e qualidade da assistência. A pesquisa teve como critérios de inclusão: artigos em português e inglês, publicados entre 2000 e 2014 e com textos completos acerca da temática em questão, sendo analisados 23 artigos. A análise dos artigos mostrou a importância da auditoria na área de saúde como instrumento a ser utilizado não somente na gestão de recursos humanos e financeiros, mas também na assistência prestada e como tal reflete positivamente nos serviços de enfermagem e em especial na saúde pública. Foi possível concluir que os dados obtidos através de processos de auditoria nos serviços de saúde são de fundamental importância na qualidade dos serviços prestados na saúde pública.

Palavras-Chave: Auditoria, Auditoria de Enfermagem, Auditoria em Saúde Pública, Qualidade da Assistência.

\section{INTRODUÇÃO}


O termo auditoria teve origem na palavra audire que, em latim, significa ouvir. Porém, também pode ser explicada através da palavra audit, de origem inglesa, que significa examinar, corrigir, certificar. Nesse sentido Pereira e Takahashi (1991) definem auditoria como sendo uma avaliação formal e sistemática de uma atividade e que indica se esta atividade está sendo realizada dentro dos objetivos propostos. Apesar de a auditoria ser utilizada por várias profissões, dentre elas podemos citar a enfermagem, trata-se de uma parte da contabilidade que objetiva avaliar a eficácia e eficiência do serviço, assim como o controle de bens patrimoniais. (REMOR, 2008).

De acordo com Santi (1988), a auditoria surgiu na área contábil entre os séculos XV e XVI, na Itália, com o objetivo de contabilizar os ganhos e gastos de um determinado negócio. Segundo Camacho e Rubin (1996) somente no século XX a auditoria foi introduzida na área de saúde objetivando avaliar a qualidade da assistência analisando os registros em prontuários. Para Pereira (2011) a auditoria em saúde trata-se de uma área de conhecimento relacionada a qualidade da assistência, trazendo benefícios as instituições no que se refere ao planejamento de utilização de recursos e insumos.

No Brasil, a auditoria de enfermagem surgiu na década de 1970. E em 2001, a auditoria de enfermagem foi aprovada através da resolução 266/01 pelo conselho federal de enfermagem (COFEN, 2001). O conhecimento sobre a prática da auditoria de enfermagem poderá melhorar a utilização dos recursos materiais e físicos disponíveis na área de saúde, o desenvolvimento pessoal, o planejamento e execução das técnicas de trabalho e o custo-benefício para quem utiliza os serviços de saúde. Quando utilizada como um processo educativo, a auditoria contribui para o gerenciamento e implantação de uma assistência de qualidade (PINTO; MELO 2010).

Segundo Santos e Merhy (2006), antes da criação do Sistema Único de Saúde (SUS) a saúde estava centrada nas ações privadas e na figura do médico. Após a sua implementação em 1988, a prioridade passou a ser a atenção básica com garantia de acesso universal e igualitário dos serviços de saúde. Garcia et. al. (2012) afirma que é importante conhecer as ações do Sistema Único de Saúde e principalmente conhecer o papel do auditor dentro das diversas atividades desenvolvidas.

Para Camelo et al., (2009), a auditoria é imprescindível para garantir o direito à saúde, por estar voltada para a avaliação da qualidade das ações de prevenção, promoção e assistência à saúde. A auditoria passou a ser primordial para a avaliação da qualidade da assistência, em especial no SUS, auxiliando na garantia ao acesso dos usuários, funcionando como instrumento de gestão e auxiliando na utilização adequada dos recursos (CAMELO et al., 2009).

Sendo assim, este artigo teve por objetivo analisar as produções científicas acerca da atuação do enfermeiro auditor na prestação de serviços na rede pública de saúde publicada em artigos no período de 2000 a 2014 e busca demonstrar o reflexo do papel do auditor na qualidade da assistência em saúde pública prestada pelo enfermeiro.

\section{METODOLOGIA}

Este estudo de natureza qualitativo-descritiva foi realizado por meio de uma revisão integrativa da literatura, sendo esta uma alternativa de pesquisa que se propõem a buscar e analisar o conhecimento já publicado sobre um determinado tema de maneira aprofundada. 
A revisão integrativa inclui a análise de pesquisas relevantes que dão suporte para a tomada de decisão e a melhoria da prática clínica, possibilitando a síntese do estado do conhecimento de um determinado assunto, além de apontar lacunas do conhecimento que precisam ser preenchidas com a realização de novos estudos. (MENDES et. al., 2008, p.123).

Para o desenvolvimento do presente artigo foi realizado uma buscas dos estudos na Biblioteca Virtual de Saúde, Base de Dados de Enfermagem (BDENF) e na Literatura Latino-Americana em Ciência de Saúde (LILACS), entre outras bibliotecas virtuais; a escolha da base se deu em função da necessidade de compreender a importância do auditor na assistência de enfermagem na rede pública. A busca foi realizada nos meses de junho a dezembro de 2014; os critérios de inclusão dos artigos definidos, inicialmente, para a presente revisão são: artigos publicados em português e inglês com textos completos disponíveis, no período compreendido entre 2000-2014. A seleção dos estudos foi realizada a partir dos seguintes descritores "Auditoria, auditoria de enfermagem, auditoria e saúde pública e qualidade da assistência”. A problemática está voltada para o questionamento sobre a falta da auditoria de enfermagem e a sua influência na qualidade da assistência na saúde pública. Foram utilizadas 23 produções sobre o tema, além de leis, decretos, manuais voltados para auditoria em saúde pública.

\section{RESULTADOS E DISCUSSÃO}

Os artigos foram analisados individualmente e de maneira sistemática levando em conta o tema abordado e dividido em quatro categorias onde foi possível evidenciar pontos importantes a cerca da história da auditoria, da atuação na auditoria de enfermagem, da auditoria no sistema único de saúde e da auditoria na qualidade da assistência prestada.

\section{UMA ABORDAGEM HISTÓRICA DA AUDITORIA}

Nesta categoria, foram incluídos os artigos que trazem uma abordagem histórica da auditoria no mundo. Com destaque para a introdução da auditoria no Brasil e em especial na área de saúde. Dos artigos analisados pode-se notar que doze deles estão voltados para uma abordagem histórica da auditoria.

Para Boynton (2002), apesar de não haver um registro preciso na história sobre o uso dos processos de auditoria, na antiguidade consta que no Egito era necessário confirmar as atividades praticadas nas grandes construções, assim como conferir os registros de arrecadação de impostos. Segundo Scarparo et al., (2010) foram encontrados registros hospitalares no Antigo Egito, o que evidencia a existência de prontuários há mais de mil anos.

Já na Grécia Antiga foram achados traços do que se assemelham aos de prontuários atuais, mas o primeiro registro de doenças foi atribuído a Hipócrates no ano de 460 antes de Cristo (a.C.). Enquanto que na Roma Antiga, auditores eram encarregados de supervisionar finanças de suas províncias. (SCARPARO et al., 2010).

A auditoria teve sua origem oficialmente em 1314, na Inglaterra, quando este país dominava os mares e as relações comerciais, através da criação do cargo de auditor do tesouro inglês. (LUZ; MARTINS; DYNEWICZ, 2007).

Oliveira e Diniz (2001) afirmam que, foi em 1956, durante a revolução industrial, com o crescimento do 
capitalismo na Inglaterra, que elementos do desenvolvimento, como por exemplo, o surgimento das grandes fábricas, colaboraram para a real necessidade de utilização das atividades da auditoria, que inicialmente era uma prática da contabilidade.

Segundo Duarte (2010) a auditoria ganhou maior notoriedade na legislação britânica, durante a Revolução Industrial, em 1956, ano considerado como de origem da auditoria do modo como é conhecida nos dias atuais. A partir de então, passou a ser utilizada em outros países, em especial, nos desenvolvidos da América do norte, que, graças ao desenvolvimento econômico, aprimoraram as técnicas de auditoria (DUARTE, 2010).

Pinto e Melo (2010) afirma que a auditoria foi introduzida na área da saúde no início do século XX, como um modo de verificar a qualidade da assistência por meio da análise dos registros em prontuários. Para Rocha (2002) esta introdução ocorreu quando o enfoque deixou de ser apenas contábil e passou a ser também administrativo buscando avaliar a eficácia e a efetividade da aplicação dos controles internos.

No Brasil, a história da auditoria no campo da saúde teve inicio no ano de 1923 na origem da previdência através da lei Eloy Chaves que criou a caixa de aposentadoria dos ferroviários, de proteção social concedendo pensão, aposentadoria, assistência médica e farmacêutica (BRASIL, 2007).

Segundo Pereira (2010), as caixas de assistência e benefícios de saúde atendiam a seus associados e dependentes conforme a categoria profissional a que pertencia o trabalhador. Em 1967 houve a união dos institutos, com o objetivo de atender a demanda na área de saúde, e com isso houve a necessidade da compra de serviços de terceiros, e o atendimento dos clientes de maneira individual organizado por classe e direito a escolha do atendimento. Deste modo, o governo adotou medidas de análise, controle e correção evitando desperdícios e cobranças indevidas, além de manter a qualidade dos serviços prestados. Com isso foi necessário a criação de um quadro de pessoas capacitadas em auditoria médica a fim de garantir o programa proposto e o funcionamento do sistema de modo integral, originando assim, o corpo funcional de auditores da previdência social (PEREIRA, 2010).

Em 1970, surgiu o Instituto Nacional de Previdência Social (INPS) e segundo Camelo (2009) a auditoria era feita apenas através da avaliação dos prontuários de pacientes e das contas hospitalares, que passaram a ser chamadas de guia de internação hospitalar (GIH) após a alta com controle formal e técnico. Ainda nos anos de 1970, foi necessário o desenvolvimento de um sistema de controle e avaliação de assistência médica, por parte do então Instituto Nacional de Previdência Social (INPS), e do Sistema Supletivo. Esta necessidade se deu em virtude do surgimento de fraudes e desvios graves, com a saída de recursos financeiros no sistema público e no sistema suplementar (PEREIRA, 2010).

Camelo et. al. (2009) ressalta que em 1978, foi instituída a secretaria de assistência médica, sujeita ao Instituto Nacional de Assistência Médica da Previdência Social (INAMPS), e após observação verificouse a necessidade de aperfeiçoamento da GIH e como isso foi criada a coordenação de controle e avaliação nas capitais e os Serviços de Medicina Social nos municípios e que segundo Ferreira et al., (2009) objetivavam a utilização dos recursos de forma racional, proporcionando o acesso a melhor alternativa de assistência a população (FERREIRA et al., 2009).

No ano de 1984, a auditoria foi instituída no Brasil através da resolução 45 de 12 de julho de 1984, pelo antigo Instituto Nacional de Assistência Médica da Previdência Social (INAMPS) que a define, segundo 
Rocha (2002) como sendo um conjunto de ações administrativas, técnicas e observacionais, que buscam a caracterização definida do desempenho assistencial, efetuado pelos integrantes de todos os níveis de execução, notadamente os referenciados às unidades médico-assistenciais próprias, contratadas, conveniadas e em regime de cogestão.

Segundo Brito e Ferreira (2006), a criação do Sistema Nacional de Auditoria (SNA) por meio da lei 8.080 de 19 de setembro de 1990 foi outro marco importante, pois veio com a função de coordenar a avaliação técnica e financeira do Sistema Único de Saúde (SUS) em todo território nacional, em colaboração com os estados, municípios e o Distrito Federal.

Pôde-se observar que ao longo da história o processo de auditoria aparece constantemente como uma importante ferramenta de gestão de recursos financeiros, e que posteriormente foi introduzida na área de saúde visando também a qualidade da assistência prestada.

Evidencia-se que no Brasil, inicialmente, a auditoria era realizada através da análise de prontuários e buscava organizar os recursos financeiros e permitir um acesso melhor aos serviços de saúde. Contudo, somente em 1984 a auditoria foi de fato introduzida no Brasil através do Instituto Nacional de assistência médica da previdência social (INAMPS, 1984).

\section{AUDITORIA EM ENFERMAGEM}

Para esta categoria foram selecionados dezesseis artigos que falam acerca da definição da auditoria de enfermagem, as finalidades das atribuições do enfermeiro auditor, as legislações que regulamentam a atuação do enfermeiro no campo da auditoria e a utilização da sistematização da assistência de enfermagem como ferramenta do processo de auditoria.

A auditoria de enfermagem, segundo Araújo (2001) é o processo pelo qual é realizada uma avaliação das atividades exercidas pela enfermagem, tratar-se de uma rigorosa investigação da qualidade da assistência de enfermagem oferecida ao paciente. Para Scarparo e Ferraz (2008) a auditoria em enfermagem busca fiscalizar o processo administrativo estabelecendo uma relação entre os objetivos traçados e e os resultados obtidos na assistência. Já para Pereira et al., (2011) a auditoria em enfermagem realiza uma função administrativa através da conferência e do controle do faturamento da conta hospitalar, verifica os procedimentos e exames realizados e, promovendo à melhoria da qualidade do serviço de enfermagem prestado através da evolução dos registros de enfermagem.

Segundo Scarparo et al., (2009), alguns autores definem a auditoria de enfermagem como sendo uma ferramenta de natureza gerencial, que tem como objetivo avaliar a qualidade da assistência, de processos e de custos. A auditoria é uma importante ferramenta para evidenciar os problemas encontrados nos prontuários, e ainda permite uma melhor avaliação e orientação para a equipe e a instituição, através de relatórios, quanto ao registro mais adequado das ações profissionais e o respaldo ético e legal, diante aos conselhos, às associações de classe e a justiça. (SETZ; D'INNOCENZO, 2009).

Os serviços de auditoria têm sido realizados em grande parte por profissionais de enfermagem, devido este demonstrar um envolvimento maior com a análise da qualidade dos cuidados oferecidos aos pacientes, sendo esta atividade regulamentada através da lei 7.498/86 que diz a respeito do exercício profissional da enfermagem e a resolução do COFEN 266/01 que dispõe sobre as atribuições do 
enfermeiro auditor. (COFEN, 2001). Além disso, o código de ética dos profissionais de enfermagem determina os princípios, deveres e proibições que orientam a conduta ética dos profissionais desta área, sendo eles auditores ou não, colaborando de maneira direta ou indireta para o processo de auditoria em enfermagem (COFEN, 2007).

Melo e Vaitsman (2008) afirmam que o enfermeiro auditor realiza as atividades de sua competência segundo os aspectos técnicos estabelecidos nas legislações vigentes do Ministério da Saúde, Agência Nacional de Saúde Suplementar, Agência Nacional de Vigilância Sanitária, Normas de auditoria de Enfermagem, código de ética de enfermagem, legislação do conselho federal de enfermagem, conselho regional de enfermagem, lei 9.656/1998, lei do código de defesa do consumidor, contratos e cobertura contratuais sempre buscando manter os padrões de qualidade.

Camelo e Silva Júnior (2006) alega que as atribuições do enfermeiro auditor estão voltadas para uma diminuição dos desperdícios, redução dos custos e a garantia de que todos os procedimentos e equipamentos utilizados e que são reembolsáveis sejam cobrados nas contas hospitalares.

A utilização dos processos de auditoria, segundo Silva et al., (2001) trás vantagens também para a equipe de enfermagem como o fortalecimento de elementos que estimulará a reflexão profissional desde que não seja utilizada como ameaça. De acordo com Kurcgant (2006), a auditoria de enfermagem não possui um caráter punitivo aos profissionais e sim uma avaliação da assistência prestada, analisando os erros para buscar fornecer base de padrões ou tendências, assim como possíveis modificações de procedimentos e técnicas administrativas.

Para Paulino (2006), a compreensão dos profissionais de enfermagem de que eles são uma das peças fundamentais de contribuição no processo de auditoria, torna a assistência ao paciente ainda mais eficiente.

Uma das ferramentas utilizadas pela auditoria em enfermagem para a avaliação da qualidade dos serviços prestados é a Sistematização da Assistência de Enfermagem (SAE) usada como um importante componente da avaliação da qualidade da assistência prestada aos pacientes (GALANTE, 2005). A SAE é utilizada como ferramenta para se ter uma auditoria de qualidade com o aperfeiçoamento da assistência prestada e ainda através da utilização de baixo custo para as instituições (BRASIL, 2011). A implementação da SAE, auxilia a comunicação do enfermeiro com a equipe multidisciplinar e ainda, segundo Setz e D'innocenzo (2009) fornece segurança ao paciente, qualidade da assistência e aumento da autonomia dos profissionais de enfermagem.

O enfermeiro auditor possui total autonomia para efetuar suas funções sem depender da presença de outros profissionais ou da autorização prévia de outro auditor, e segundo Faraco e Albuquerque (2004) também tem o direito ao acesso de toda e qualquer documentação e realização de visitas e entrevistas a pacientes, sendo a sua principal função a avaliação do processo de qualidade, de deficiência no atendimento, planejamento e satisfação do usuário dos serviços de saúde.

A auditoria de enfermagem possui todos os respaldos legais que contribuem para que esta seja uma área crescente com muitas perspectivas nos próximos anos, mesmo que atualmente poucos profissionais atuem nesta área, sendo ainda necessária uma melhor orientação dos enfermeiros a cerca da importância da auditoria no campo da enfermagem assim como os benefícios que a auditoria traz a este serviço (BERTI; 
ALMEIDA, 2005).

No que se refere a auditoria de enfermagem, os autores apontam que esta não avalia somente os recursos materiais utilizados na assistência como também a qualidade dos serviços prestados. Pode-se observar que de um modo geral, a auditoria é realizada por profissionais de enfermagem por estes prestarem uma assistência mais direta ao paciente sendo regularizada pela lei do exercício profissional e código de ética de enfermagem, entre outras legislações (BERTI; ALMEIDA, 2005).

É possível destacar ainda que a auditoria se mostra importante também para o desenvolvimento profissional uma vez que proporciona uma reflexão acerca da qualidade da assistência prestada, não sendo em nenhuma hipótese uma medida de caráter punitivo.

\section{AUDITORIA NO SISTEMA ÚNICO DE SAÚDE}

Para a composição desta categoria foram utilizados 12 artigos que tratam da utilização da auditoria no Sistema Único de saúde através da lei Orgânica de Saúde, e as leis que regulamentam o Sistema Nacional de Auditoria (SNA) e a Agência Nacional de Saúde Suplementar (ANS) (OLIVEIRA, 2009).

A auditoria no Sistema Único de Saúde (SUS) objetiva auxiliar na qualidade da assistência à saúde fornecida à população na busca da efetivação da universalidade, equidade e integralidade, sendo estes princípios fundamentais do SUS, além do princípio da economicidade na utilização dos recursos financeiros envolvidos, de acordo com Klustchovski (2009 apud OLIVEIRA, 2009).

A lei no 8.080 de 19 de dezembro de 1990 regula as ações e serviço de saúde em todo território nacional. (BRASIL, 1990) Essa lei estabelece o Sistema Nacional de Auditoria (SNA) como um meio de controle técnico e financeiro sob competência do Sistema Único de Saúde e em colaboração com os Estados, municípios e o Distrito Federal. Porém, foi em 27 de julho de 1993 que o SNA foi instituído, através do art. $6^{\circ}$ da Lei n. 8.689 (BRASIL, 1993) e tendo a sua regulamentação através do decreto n. 1.651 de 28 de setembro de 1995 (BRASIL, 1995).

Regulamentada através da Lei n. 9.961/2000, a Agência Nacional de Saúde Suplementar (ANS) busca controlar as ações e serviços de saúde das operadoras e de outros órgãos públicos (BRASIL, 2000) com o objetivo de manter a qualidade da assistência à saúde.

O SUS define como objetivo a transformação do modelo de assistência à saúde, afastando a saúde da condição de mercadoria estabelecendo-a como um direito de cidadania. A consolidação do SUS implica em universalização, acesso, qualidade dos serviços e controle social. Por sua vez, o SNA foi regulamentado objetivando o controle sistemático da estrutura e processos e dos resultados no contexto do SUS (MELO e VAITSMAN, 2008).

Um grande desafio para as instituições públicas têm sido desenvolver medidas para garantir cada vez mais a melhoria da qualidade da assistência. A auditoria permite criar subsídios para elaborar novos propostas e métodos de gerenciamento das atividades e recursos ligados à assistência (CUNHA et al., 2003).

De acordo com Camelo et al., (2009), a descentralização e a integração dos serviços de auditoria são 
alguns dos princípios e diretrizes estabelecidos pelo Departamento Nacional de Auditoria com o objetivo de garantir a atuação em todo o território nacional, com as suas tarefas específicas divididas e definidas para cada esfera de gestão do SUS.

Há muita responsabilidade e interesses quando se audita na área de saúde, pois o trabalho no SUS é de alta complexidade, devido à necessidade em manipular cuidadosamente uma grande quantidade de informações extraídas, analisadas e interpretadas (BERTI; ALMEIDA, 2005).

A avaliação dos processos de auditoria, segundo Vaitsman e Andrade (2005), através do planejamento da atenção em saúde, possibilita a organização dos serviços de saúde de forma mais humanizada. No SUS, a auditoria em saúde busca gerenciar e controlar os recursos financeiros públicos com ações voltadas para a atenção continuada e acompanhamento da qualidade da assistência colaborando com humanização das ações de saúde dentro dos recursos financeiros disponíveis no SUS (SCARPARO, 2010).

Santos e Merhy (2006) ressalta que uma das finalidades da auditoria está voltada para a avaliação contábil que auxilia a gerência no que diz respeito ao controle financeiro dos recursos públicos. Dessa forma, a avaliação e o controle dos recursos financeiro na saúde pública, são importantes instrumentos de monitoramento das políticas públicas implantadas, com o propósito de estabelecer objetivos da instituição, a recondução dos recursos e ações em saúde de modo resolutivo e centralizado na equidade (SANTOS; MERHY, 2006).

Por ser um sistema de gestão, Camelo et. al. (2009) afirma que a auditoria fortalece o SUS e atua como uma importante ferramenta gerencial através do planejamento das práticas assistenciais, organizando e fiscalizando a assistência em saúde dentro dos parâmetros financeiros. Desta forma, a realização da auditoria propicia a avaliação da assistência em saúde fornecida aos usuários do SUS.

Os artigos revisados mostraram que a auditoria, dentro do contexto da saúde pública, busca pôr em prática os princípios do Sistema Único de Saúde (SUS) visando principalmente a qualidade da assistência no setor público.

A auditoria no SUS também permite gerenciar os recursos financeiros públicos o que influencia diretamente em uma melhor implementação de políticas públicas e consequentemente na qualidade da assistência prestada.

\section{AUDITORIA E QUALIDADE DA ASSISTÊNCIA}

Nesta categoria é possível observar a importância da auditoria de enfermagem na qualidade da assistência prestada no que diz respeito a auditoria como forma de avaliação da qualidade, eficácia e eficiência dos serviços oferecidos através da assistência. Foram utilizados 10 artigos para compor esta categoria.

Para o Ministério da Saúde (2011) a qualidade da assistência à saúde busca reduzir os riscos, elevar o grau de competência profissional, ser eficaz no uso dos serviços, visando à satisfação do cliente associando a um efeito favorável na promoção da saúde.

$\mathrm{Na}$ busca pela qualidade da assistência aos usuários da saúde, os padrões são estabelecidos gradativamente por meio de estudos realizados na própria instituição, segundo Abdon et al., (2009). Desta 
forma, a auditoria surge como um meio de avaliação da qualidade da assistência em saúde de uma determinada população. De acordo com Camelo et al., (2009) a auditoria é uma ferramenta fundamental para mensurar a qualidade da assistência, acrescentando valores financeiros e qualitativos, proporcionando formas para o desenvolvimento das atividades em saúde, visando melhorar a assistência. Deste modo, a auditoria funciona como um método de avaliação sistemática de um processo ou de uma atividade, propiciando informações para averiguação da qualidade do serviço de saúde oferecido por uma instituição.

No Brasil, segundo Scarparo e Ferraz (2008), existe uma ideia errônea de que a auditoria em saúde está ligada somente a atividades burocráticas, voltada para a área contábil e financeira. Agora nota-se que a auditoria é uma aliada da eficiência e eficácia dos serviços prestados e da satisfação do cliente.

O empenho em garantir a melhoria da qualidade da assistência prestada, o desenvolvimento de novas propostas e métodos que possibilitem os processos de trabalho e os recursos voltados à assistência tem sido um grande desafio para os serviços de enfermagem. Neste contexto, a auditoria contribui, de forma restrita com as funções burocráticas e administrativas, porém, nota-se uma mudança gradativa com o foco para a assistência. $\mathrm{Na}$ área de enfermagem, a auditoria possibilita o desenvolvimento de indicadores em saúde, permitindo o desenvolvimento de critérios de avaliação e formação de novos conhecimentos, o que permite avaliar os problemas enfrentados pela enfermagem, as condutas estabelecidas para cada um deles e a solução proposta para cada problema (SCARPARO et al., 2010).

$\mathrm{Na}$ área de saúde, quando se trata de qualidade, Camelo et al., (2009) afirma que devemos estar atentos ao nível de satisfação dos clientes, os fatores variáveis no ambiente da área de saúde e os possíveis efeitos na credibilidade da instituição. Por esta razão, alguns serviços da área de saúde estão fazendo uso da auditoria, como meio de melhoria continuada da qualidade da assistência prestada. Com o objetivo de diminuir os erros, visando à qualidade da assistência, os serviços de saúde fazem uso da auditoria por meio da fiscalização dos serviços e procedimentos, da auditoria de contas, em beneficio da promoção da qualidade à saúde do paciente (MELO; VAITSMAN, 2008).

De acordo com Faraco e Albuquerque (2004), a auditoria oferece subsídios aos profissionais de enfermagem para reorientar suas atividades. Para Mota (2003) o processo da auditoria de enfermagem deve ter objetivos claros que evidencie os pontos fracos do serviço e afirma ainda que ao longo dos anos, a auditoria de enfermagem vem ganhando novas dimensões e revelando a sua importância dentro das instituições.

Segundo Riolino e Kliukas (2003), a enfermagem precisa de profissionais competentes e preparados que possam lhe dar com os avanços da área de saúde, pois está diretamente ligada com a qualidade da assistência por ser responsável pelos cuidados. Na busca pela qualidade da assistência, a competência é a habilidade de desenvolver funções buscando primeiramente a qualidade do serviço.

Galante (2005) afirma que a prática da auditoria de enfermagem resulta em benefícios aos pacientes, os quais poderão usufruir de serviços oferecidos com maior eficiência e consequentemente uma assistência de melhor qualidade. Estes benefícios não se restringem apenas aos usuários dos serviços, mas também aos que prestam assistência, como por exemplo, a equipe de enfermagem. Ao revisarem as atividades desempenhadas, conseguirão resultados com sucesso nos procedimentos futuros, levando os profissionais a refletirem, levando a um maior interesse na área científica, estabelecendo elementos para uma 
assistência de qualidade cada vez melhor.

Os estudos aqui revisados mostram uma relação direta entre o processo de auditoria e a qualidade da assistência prestada. Apesar de inicialmente o trabalho em auditoria estar voltado para a área burocrática e administrativa pode-se concluir que a mesma influencia diretamente na área assistencial.

Nessas pesquisas, os autores ressaltam que para que a auditoria seja utilizada de maneira eficaz é necessário que os profissionais de saúde se conscientizem da importância da utilização da auditoria na área da saúde e da qualificação dos profissionais que atuam na auditoria.

\section{CONSIDERAÇÕES FINAIS}

O presente estudo permitiu analisar a importância da auditoria ao longo dos anos, que com o passar do tempo deixou de ser exclusivamente um ramo da contabilidade e passou a ser utilizada por outras profissões, dentre elas a enfermagem, aplicada na área de saúde, com uma visão voltada não somente para o controle de gastos e prejuízos financeiros, mas principalmente preocupados como uma assistência de qualidade.

A auditoria em saúde é uma área que exige um alto grau de qualificação do profissional, uma vez que as ações desempenhadas na assistência a saúde requer muita responsabilidade, além de fornecer os elementos necessários para orientar as atividades desempenhadas pelos auditores.

$\mathrm{Na}$ área da saúde, o enfermeiro é o profissional melhor qualificado para desempenhar e executar as ações de auditoria, pois está diretamente ligado a assistência ao paciente. Este profissional, no desempenho de suas atividades em auditoria, deve buscar reduzir os gastos, evitar desperdícios e assegurar uma assistência de qualidade.

Na saúde pública, a auditoria funciona como uma importante ferramenta gerencial, que quando utilizada corretamente fornece subsídios ao sistema único de saúde (SUS) no sentido de planejar, organizar e fiscalizar melhor as práticas assistenciais, avaliando a melhor aplicação dos recursos públicos e principalmente a qualidade da assistência fornecida aos usuários, assegurando assim os princípios fundamentais da universalidade, equidade e integralidade garantidos por lei e promovendo a saúde que de acordo com a constituição federal é um direito de todos e dever do Estado fornecê-la.

Assim, podemos concluir que na área de saúde a auditoria de enfermagem tem um papel fundamental na avaliação da qualidade da assistência, da eficácia e da efetividade dos serviços prestados, além da avaliação e controle dos recursos financeiros, sendo estes elementos essenciais para a construção de uma saúde pública de qualidade garantindo aos usuários do sistema único de saúde acesso universal e humanizado.

\section{REFERÊNCIAS}

ABDON, Jéssica Bentes; DODT, Regina Cláudia Melo; VIEIRA, Danielly Pontes; MARTINHO, Neudson Johnson; CARNEIRO, Elioneide Paulo; XIMENES, Lorena Barbosa. Auditoria dos Registros na Consulta de Enfermagem acompanhando o Crescimento e Desenvolvimento Infantil. Revista da Rede de Enfermagem do Nordeste. Fortaleza, v. 10, n. 3, p. 90-96, jul./set.2009. 
ARAUJO, Inaldo da Paixão Santos. Introdução à auditoria operacional. 2.ed. Rio de Janeiro: FGV, 2001.

BOYNTON, William C.; JOHNSON, Raymond N.; KELL, Walter G. Auditoria: tradução autorizada. 7 ed. São Paulo: Atlas; 2002.

BRASIL. Ministério da Saúde. Lei n. 8.080, de 19 de setembro de 1990. Dispõe sobre as condições para promoção, proteção e recuperação da saúde, a organização e funcionamento dos serviços correspondentes e dá outras providências. Diário Oficial da União, Brasília, DF, n. 182, Seção I, p. 18.055, 20 set. 1990.

Diário Oficial da república Federativa do Brasil. Lei n. 8.689, de 27 de julho de 1993. Dispõe sobre a extinção do Instituto Nacional de Assistência Médica da Previdência Social (INAMPS) e dá outras providências. Brasília, DF, p. 10.573, 28 jul. 1993b. Disponível em: http://www.planalto.gov.br/ccivil_03/leis/L8689.htm. Acesso em: 19 jan. 2015

Senado Federal. Decreto n. 1.651, de 28 de setembro de 1995. Regulamenta o Sistema Nacional de Auditoria no âmbito do SUS. Diário Oficial da União, Brasília, DF, 28 set. 1995. Disponível em: <http://www.t.senado.gov.br/decreto1651.html> Acesso em: 19 jan. 2015.

Diário Oficial da república Federativa do Brasil. Lei n. 9.961 de 28 de janeiro de 2000. Cria a Agência Nacional de Saúde Suplementar - ANS e dá outras providências. Brasília, DF, 28 jan. 2000. Disponível em: http://www.planalto.gov.br/ccivil_03/Leis/L9961.htm. Acesso em: 19 jan. 2015

Ministério da previdência. Panorama da previdência social brasileira. $2^{\mathrm{a}}$ Ed. Brasília: MPS, SPS, SPC, ACS. 2007. Disponível em: http://www.inss.gov.br/arquivos/office/3_090126-092058-729.pdf. Acesoo em: 21 mai. 2015

Ministério da Saúde. Auditoria do SUS: orientações básicas. Secretaria de Gestão Estratégica e Participativa. Sistema Nacional de Auditoria. Departamento Nacional de Auditoria do SUS. Brasília (DF), 2011.p.48. Série A. Normas e Manuais Técnicos.

BERTI, H.W; ALMEIDA, E.S. Percepção dos gestores municipais de saúde sobre qualidade da assistência e controle social. Revista nursing, São Paulo, v. 8, n. 88, p. 425-431, set.2005.

BRITO, Magnólia Fernandes; FERREIRA, Leonardo Nunes. A importância da auditoria interna hospitalar na gestão estratégica dos custos hospitalares. 2006 (trabalho de conclusão de curso). Universidade Católica de Brasília; Goiás (GO): 2006.

CAMACHO, Luiz Antônio Bastos, RUBIN, Haya Rahel. Reliability of medical audit in quality assessment of medical care. Cad. Saúde pública: Rio de janeiro, V. 12, n. 2, p. 85-93,1996

CAMELO, Tatiana Vasques.; SILVA JUNIOR, Osnir.Claudiano. Tratamento do tema auditoria de enfermagem em base de dados. Revista Meio ambiente saúde. Manhuaçu (MG), v. 1, n. 1, p. 7-12, 2006.

CAMELO, Silvia Helena Henriques; PINHEIRO, Aline; CAMPOS, Domitila; OLIVEIRA, Tatiana Lentz de. Auditoria de enfermagem e a qualidade da assistência à saúde: uma revisão da literatura. Revista eletrônica de enfermagem. Ribeirão Preto: v. 14, n. 4, p. 1018-1025, 2009. 
COFEN. Conselho Federal de Enfermagem. Resolução 266, de 25 de outubro de 2001. Dispõe sobre as atividades do enfermeiro auditor. Brasília; 2001. Disponível em: <http://www.novo.portalcofen.gov.br/resoluo-cofen-2662001_4303.html> Acesso em: 05 fev.2015

Conselho Federal de Enfermagem. Código de ética dos profissionais de enfermagem. Rio de Janeiro, 8 fev. 2007. Disponível em: http://se.corens.portalcofen.gov.br/codigo-de-etica-resolucaocofen-3112007. Acesso em: 05 fev. 2015

COSTA, Maria Suêuda; FORTE, Benedita Pessoa; ALVES, Maria Dalva Santos; VIANA, Jamille Forte; ORIÁ, Mônica Oliveira Batista. Auditoria em enfermagem como estratégia de um marketing profissional. Revista Brasileira de Enfermagem. Brasília: v. 57, n. 4, p. 497-499, jul/ago. 2004.

CUNHA, Ana Paula; OROFINO, Cláudia Luiza Fonseca; COSTA, Arigelinda Pereira da; JÚNIOR, Giuseppe Donato. Serviços de enfermagem: um passo decisivo para a qualidade. Revista Nursing. São Paulo: v. 6, n. 60, p. 25-30, mai. 2003.

DUARTE, Lara Raquel Santos. Auditoria financeira. 2010. Dissertação (Mestrado em Gestão), Faculdade de Economia da Universidade de Coimbra. Coimbra, 2010.

FARACO, Michel Maximiano. DE ALBUQUERQUE, Gelson Luiz. Auditoria do método de assistência de enfermagem. Revista brasileira de enfermagem, Brasília, v. 57, n. 4, p. 302-305. Jul/ago. 2004.

FERREIRA, Tânia S.; SOUZA-BRAGA, André Luiz de; CAVALCANTI-VALENTE, Geilsa Soraia; SOUZA, Deise Ferreira de; CARVALHO-ALVES, Enilda Moreira.

Auditoria em enfermagem: o impacto das anotações de enfermagem no contexto das glosas hospitalares. Revista Aquichan, v. 9, n. 1, abril, 2009, p. 38-49.

GARCIA, Emerson Gomes et al. A auditoria e o enfermeiro como ferramentas de aperfeiçoamento do SUS. Revista Baiana de saúde pública. Salvador V.36, n.2, p.539-559. Abr/jun. 2012.

GALANTE, A.C. Auditoria hospitalar do serviço de enfermagem. Goiânia: AB, 2005.

KURCGANT, P. Administração em enfermagem. São Paulo: EPU, 2006

LUZ, Alessandra; MARTINS, Andréia Perreira, DYNEWICZ, Ana Maria. Características de anotações de enfermagem encontra das em auditoria. Revista Eletrônica de Enfermagem [Internet], v. 9. n. 2, p. 344-361, Mai-Ago 2007.

MELO, Marilene Barros de; VAITSMAN, Jeni. Auditoria e avaliação no sistema único de saúde. Revista São Paulo em perspectiva. São Paulo, v. 22, n. 1, p. 152-162, jun., 2008.

MENDES, Karina Dal Sasso; SILVEIRA, Renata Cristina de Campos Pereira; GALVAO, Cristina Maria. Revisão integrativa: método de pesquisa para a incorporação de evidências na saúde e na enfermagem. Texto contexto - enfermagem, Florianópolis: v. 17, n. 4, Dez. 2008. 
MOTTA, Ana Leticia Carnevalli. Auditoria de Enfermagem nos hospitais e seguradoras de saúde. São Paulo: Látria; 2003.

OLIVEIRA, Luiz Martins de; DINIZ FILHO, André. Curso básico de auditoria. São Paulo: Atlas, 2001.

OLIVEIRA, Valter Luiz de. Relato histórico da auditoria e sua aplicação no sus e nas operadoras de saúde suplementar. 2009, 37 f. Monografia (pós-graduação em Auditoria e gestão em saúde), Universidade educacional Tuiuti do Paraná. Curitiba, 2009

PAULINO, Emmanuela de Almeida. Conhecimento dos enfermeiros acerca da auditoria hospitalar. João Pessoa: Atlas, 2006.

PEREIRA, Samira Auxiliadora; MIRANDA, Núbia Rodrigues Ramos; COSTA, Regiane Ferreira. Auditoria em enfermagem e suas interfaces com o cuidado: uma revisão de literatura. Percurso acadêmico, Belo Horizonte, v. 1, n. 2, p. 287-306, jul/dez 2011

PEREIRA, LL. TAKAHASHI, RT. Auditoria em enfermagem. In.: Kurcgant P, organizadora. Administração em enfermagem. São Paulo: EPU, 1991. P. 215-222.

PEREIRA, Adriana Aparecida. O papel do enfermeiro auditor na instituição hospitalar e no sistema de saúde suplementar. 2010. Monografia (Pós-graduação em Auditoria e Gestão em Saúde). Universidade Tuiuti do Paraná, Curitiba, 2010.

PINTO, Karina Araújo; MELO, Maria Meira de. A prática da enfermeira em auditoria em saúde. Revista escola de enfermagem USP. São Paulo, Vol.44, n. 3, Set. 2010.

REMOR, Lourdes de Costa. Auditoria do SUS em Santa Catarina. Revista de Saúde Pública. Santa Catarina, Forianópolis: v.1, n.1, p.71-83. jan/jun.2008.

RIOLINO, Angelina Nagy; KLIUKAS, Gabriela Brunetti Vellego. Relato de experiência de enfermeiras no campo da auditoria de prontuários: uma ação inovadora. Revista Nursing. São Paulo, v. 65, n. 6, p. 35-39, out. 2003.

ROCHA, Enock Emmanoel Moreira; SILVEIRA FILHO, Ilbanez Bonfim; SANT'ANNA, Tomé Roberto Ferreira. A importância da auditoria no sistema único de saúde - SUS. 2002 (trabalho de conclusão de curso). Universidade Federal da Bahia; Salvador 2002.

SANTI, Paulo Adolpho. Introdução à auditoria. São Paulo: atlas; 1988.

SANTOS, Fausto Pereira dos. MERHY, Emerson Elias. A regulação pública da saúde no estado brasileiro - Uma revisão. Revista Interface - comunicação, saúde, educação. São Paulo, v.10, n.19, p.25-41, jan/jun 2006.

SCARPARO, Ariane Fazollo. FERRAZ, Clarice Aparecida. Auditoria de enfermagem - Identificando suas concepções e métodos. Revista Brasileira de Enfermagem. Brasília: v. 61, n. 3, p.302-305, jun. 2008. 
SCARPARO, Ariane Fazzolo; FERRAZ, Clarice Aparecida; CHAVES, Lucieli Dias Pedreschi; ROTTA, Carmen Silva Gabriel. Abordagem conceitual de métodos e finalidade da auditoria de enfermagem. Revista Rede de enfermagem do nordeste. Fortaleza: v.10, n. 1, p.124-130, 2009.

SCARPARO, Ariane Fazzolo; FERRAZ, Clarice Aparecida; CHAVES, Lucieli Dias Pedreschi e GABRIEL, Carmen Silva.Tendências da função do enfermeiro auditor no mercado em saúde. Texto contexto enfermagem, Florianópolis: v. 19, n. 1, 85-92. jan/mar 2010

SETZ, Vanessa Grespan; D'INNOCENZO, Maria. Avaliação da qualidade dos registros de enfermagem no prontuário por meio da auditoria. Acta paulista de enfermagem, São Paulo: v. 22, n. 3, p. 313-17, fev. 2009.

SILVA, F.P. Silva FP, Silva MAG. Conceição, SM. Nascimento, EJS. Auditoria em enfermagem: burocracia ou necessidade. Porto Alegre: Universidade Federal do rio Grande do Sul; 2001

VAITSMAN, Jeni.; ANDRADE, Gabriela Rieveres Borges. Satisfação e responsabilidade: formas de medir a qualidade e a humanização da assistência à saúde. Ciência Saúde coletiva. Rio de Janeiro: v. 10, n. 3, Jul/Set 2005.

${ }^{[1]}$ Enfermeira Pós graduada em Gestão e Auditoria em Serviços de Saúde pela Faculdade Tecnológica Machado de Assis - FAMA.

${ }^{[2]}$ Bióloga. Doutora em Ciências Biológicas - Área de Concentração Genética. Professora e Pesquisadora no CESUPA - Centro Universitário do Estado do Pará.

${ }^{[3]}$ Psicóloga. Mestre em Teoria e Pesquisa do Comportamento. Doutoranda em Doenças Tropicais pelo Núcleo de Medicina Tropical da UFPA (NMT-UFPA). Docente da Universidade do Estado do Pará UEPA.

[4] Biomédica. Doutora em Doenças Tropicais. Docente e Pesquisadora da Universidade Federal do Amapá, AP. Pesquisador colaboradora do Núcleo de Medicina Tropical da UFPA (NMT-UFPA).

${ }^{[5]}$ Biólogo. Doutor em Teoria e Pesquisa do Comportamento. Docente e Pesquisador do Instituto Federal do Amapá - IFAP.

${ }^{[6]}$ Doutora em Psicanálise Clínica, Pesquisadora pelo Centro de Pesquisa e Estudos Avançados.

${ }^{[7]}$ Registered Nurse e Bachelors in Nursing Science. USA - California

${ }^{[8]}$ Biólogo. Doutor em Medicina/Doenças Tropicais. Docente e Pesquisador na Universidade Federal do Pará - UFPA. Pesquisador no Laboratório de Toxicologia Humana e Ambiental e no Laboratório de Estresse Oxidativo do Núcleo de Medicina Tropical da UFPA (NMT-UFPA).

\section{PUBLIQUE SEU ARTIGO CIENTÍFICO EM:}

https://www.nucleodoconhecimento.com.br/enviar-artigo-cientifico-para-submissao 